\title{
Strength characterization of adhesive joint surface treated by large-area EB irradiation
}

\author{
Yuya OMIYA*, Kenji TOYOTA*, Ryota NAKANISHI**, Togo SHINONAGA*, \\ Tadashi SHIOTA*, Akira OKADA* and Masahiro FUJI* \\ *Graduate school of Natural Science and Technology, Okayama University \\ 3-1-1 Tsushima-naka, Kita-ku, Okayama, 700-8530, Japan \\ E-mail: omiya@okayama-u.ac.jp \\ ${ }^{* *}$ Industrial Technology Center of Okayama Prefecture \\ 5301 Haga, Kita-ku, Okayama, 701-1296, Japan
}

Received: 9 December 2020; Revised: 22 January 2021; Accepted: 18 February 2021

\begin{abstract}
With recent improvements in adhesive performance, adhesive joints are being increasingly used in various industrial fields. Despite their advantage of joining dissimilar adherends, adhesive joints are considered unsuitable for key components of mechanical structures due to their large variation in joint strengths. There are many factors that affect the strength of adhesive joints. However, the general bonding mechanisms are (1) mechanical, (2) physical, and (3) chemical bonding. The adhesion mechanism is complex because these phenomena occur simultaneously. Thus, it is necessary to consider these factors separately. Moreover, it is important to understand the bonding mechanism of interfaces in adhesive joints and to improve the reliability of the joint strength. In this study, the tensile strengths of single lap joints were measured to investigate the influence of large-area EB irradiation on strength characterization of adhesive joints. Aluminum alloy plates (A6061) were used as the adherend. Other surface treatments, which were sanding by emery paper, shot blast, and plasma irradiation, were also conducted for comparisons. Each treated surface of the adhered was examined with respect to the surface profile, wettability, and surface analysis by XPS. Results showed that the wettability of treated surfaces of adherend improved, and tensile strength of surface treated adhesive joints was also improved compared with that of the non-treated specimen. Specifically, the strength of the joint with EB irradiation was 1.9 times larger than that of the non-treated specimen. Finally, the effect of large-area EB irradiation on adhesive characterization was discussed.
\end{abstract}

Keywords : Adhesive joint, EB irradiation, Large-area, Joint strength, Wettability, Surface profile

\section{Introduction}

Recently, adhesive joints have been increasingly used in automobile and aircraft structures. Many studies on the strength of these joints subjected to loading can be referred to here (da Silva and Adams, 2007; Sawa et al., 2000) However, the reliability of strength under loading is yet to be assessed. As it is desirable for adhesive joints in the principal parts of mechanical structures to have sufficient strength and reliability against high loading rates, investigating a method for improved joint strength and reliability is crucial.

In this regard, surface treatment has been used to improve the strength and reliability of adhesive joints. As a simple method of this, the method (Sakata et al., 1998; Harris and Beevers, 1999; Ghumatkar et al., 2016; Misukure et al., 2019) of changes in surface roughness has been widely used. The surface treatment focused on the wettability of the adhesion surface (Molitor, 2001). However, the mechanism for improving the adhesive strength has not been sufficiently discussed. Although many factors affect the strength of adhesive joints, it is dependent on mainly (1) mechanical, (2) physical, and (3) chemical bonding. The adhesion mechanism is complex because these phenomena occur simultaneously. Thus, it is necessary to consider these factors separately. 
As a surface treatment method, the large-area electron beam (EB) irradiation method was used (Uno et al., 2005; Shinonaga, 2018). This method made it possible to irradiate an unfocused electron beam having a sufficient energy density to melt the metal surface. A previous study showed that the wettability and corrosion resistance of mold steel were improved by EB irradiation due to the formation of a thin resolidified layer with fine micro-structures. Therefore, the workpiece surface can be expected to become activated by the EB irradiation, and the resulting EB irradiated surface would affect the adhesive strength. However, applications of this method to the adhesive field have not yet been discussed.

In this study, we investigated the influence of large-area EB irradiation on the strength characterization of adhesive joints. The changes in the surface of the adherend were clarified by changing the EB irradiation conditions. The surface profile and wettability (contact angle of water) were studied, and adherend surface analysis was performed using X-ray photoelectron spectroscopy (XPS) to characterize the strength of the adhesive joint. Tensile tests with a single lap adhesive joint were performed for assessing the joint strength. To examine the effect of changes in surface roughness, two surface treatments, sanding by emery paper and shot blast, were conducted. Moreover, to discuss the surface activity, plasma was used for the surface treatment. Aluminum alloy plates (A6061) were used as the specimens. A one pack type thermo-curing adhesive and an epoxide-based adhesive were used.

\section{Tensile tests}

\subsection{Test specimen and test method}

To measure the strength of the adhesive joint, the tensile tests were carried out using the adhesive lap joints. Figure 1 shows the dimensions of the test specimen (adhesive lap joints), made of A6061, for the tensile tests, which was obtained by milling. The adhesive was a one pack type thermo-curing adhesive and an epoxide-based adhesive. The adhesion area was $10 \times 10 \mathrm{~mm}^{2}$ at the end of the adherend plate, and the thickness of adhesion was $0.15 \mathrm{~mm}$. The curing temperature and time of this adhesive were $120^{\circ} \mathrm{C}$ and $2 \mathrm{~h}$, respectively.

Four types of surface treatments (i.e., sanding by emery paper, shot blast, plasma irradiation, and large-area EB irradiation) were applied to the adhesion area.

Figure 2 shows the schematic of the test apparatus. A tensile loading was applied to the test specimens using a tensile testing machine. To prevent the eccentricity of the tensile direction, the test specimen was fixed with a universal joint along with spacers with plate thickness of $2 \mathrm{~mm}$.

\subsection{Surface treatments}

As mentioned above, large-area EB irradiation (EB), sanding by emery paper (EP), shot blast (SB), and plasma irradiation (plasma) were used as surface treatments, conducted after cleaning the surface with acetone. A specimen in which the adhesion area was cleaned with acetone only was called "non-treated." The strengths of the specimens with four types of surface treatments, especially large-area EB irradiation (EB), were compared with that of the non-treated specimen to investigate the influence of these treatments.

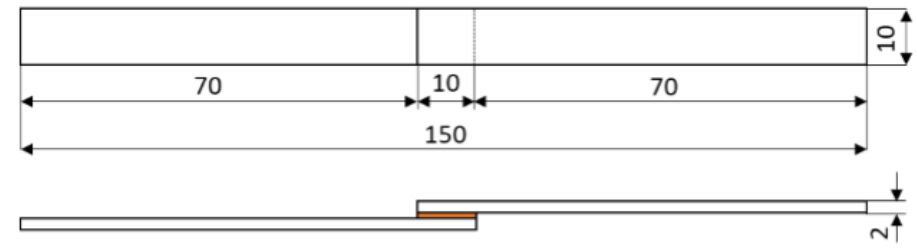

Fig. 1 Dimensions of the test specimen (unit: $\mathrm{mm}$ )

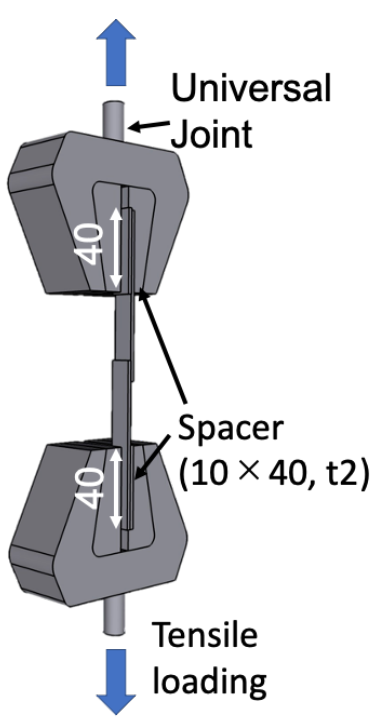

Fig. 2 Schematics of the test setup for measuring the joint strength (unit: mm) 
Figure 3 shows the schematic diagram of the EB irradiation apparatus, which can melt and vaporize the adherend surface by EB irradiation. In this EB irradiation process, changes in surface roughness and removal of the oxide film on the adherend surface can be expected. Table 1 shows the EB irradiation treatment conditions used in this study. To investigate the influences of the energy density and shot number, different conditions of 5, 10, and $15 \mathrm{~J} / \mathrm{cm}^{2}$, and 10,20 , and 30 shots, respectively, were used. To investigate the influence of the surface roughness on the joint strength, EP and SB specimens were used. The EP specimen was sanded using emery paper \#240, while the SB specimen was shot with alumina powder of particle sizes of \#30, \#60, \#120, and \#220. To apply chemical modification without changing the surface roughness, a plasma specimen was used, which was treated with an atmospheric plasma treatment device. The distance of plasma irradiation from the adherend surface was $1 \mathrm{~mm}$, and the feeding speed of stage was $2.5 \mathrm{~m} / \mathrm{s}$. The adherend plate was heated using a heater during plasma irradiation.

EP and SB specimens were subjected to ultrasonic cleaning with acetone before bonding, while the EB and plasma specimens were adhered within 10 min after these irradiations.

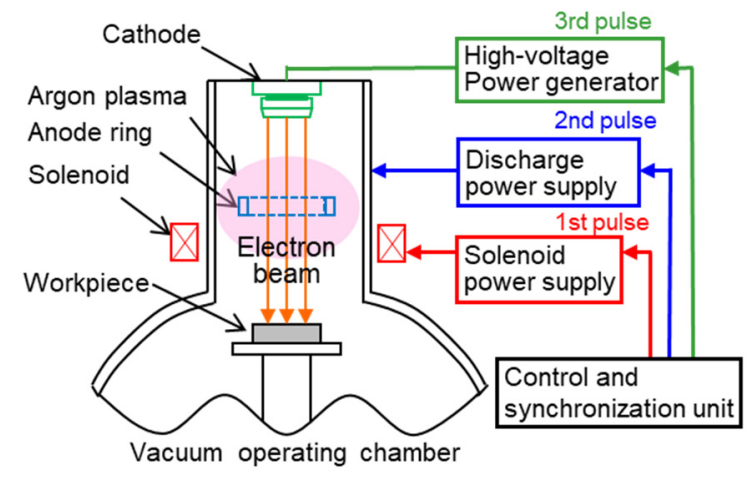

Table 1 Large-area EB irradiation conditions

\begin{tabular}{cc|c}
\hline Energy density & {$\left[\mathrm{J} / \mathrm{cm}^{2}\right]$} & $5,10,15$ \\
\hline Shot number & {$[$ shots $]$} & $10,20,30$ \\
\hline Pulse duration & {$[\mu \mathrm{s}]$} & 2.0 \\
\hline Pulse frequency & {$[\mathrm{Hz}]$} & 0.125 \\
\hline
\end{tabular}

Fig. 3 Schematic diagram of large-area EB irradiation apparatus

\subsection{Laser microscope images and surface profile of adherend surface after the treatments}

The surface state of the adherend must be assessed to discuss the strength of the adhesive joint. The surfaces were observed, and their roughness levels were measured using a laser microscope.

Figure 4 shows a laser micro-image of the non-treated specimen. The surface roughness $S a$ of the non-treated surface was $0.256 \mu \mathrm{m}$. Machining marks were observed on the surface due to milling. Figure 5 shows the laser micro-images of the surface treated by large-area EB irradiation under different conditions of shots and energy density. The surface roughness is seen to increase with increasing shot number, except for the condition where the energy density was 15 $\mathrm{J} / \mathrm{cm}^{2}$. Changes in the waviness of the EB irradiated surface were observed from profile curves. These results suggest that the solid solution elements in aluminum alloys such as silicon are preferentially melted and/or evaporated by the EB irradiation.

Figures 6 and 7 show the laser micro-images of the surfaces treated by emery paper and that treated by shot blast, respectively. The surface roughness was found to increase, and the characterization of the treated surfaces was completely random. The surface and roughness of the surface treated by plasma were the same as those of the non-treated specimen.

\subsection{Surface analysis by XPS}

Table 2 shows the atomic concentration of the adherend surface after surface treatment measured by XPS. C, O, and $\mathrm{Al}$, which were the main atoms on the aluminum alloy surface, are discussed here. The atomic concentrations of EP and SB surfaces were not significantly different compared with the non-treated surface. Thus, the measured result of the nontreated surface was indicated as a representative value. A higher $\mathrm{C}$ due to organic matter, such as acetone, was observed on the non-treated surface, which was attributed to the cleaning with acetone before the bonding process in non-treated, EP, and SB surfaces. On the contrary, the amount of C on the EB and plasma surfaces was less than that on the nontreated surface. It was considered that the oxide film on the adherend surface was removed due to evaporation on the EB surface. On the plasma-treated surface, the plasma reacted with organic matter on the adherend surface, and $\mathrm{C}$ was removed. 
Omiya, Toyota, Nakanishi, Shinonaga, Shiota, Okada and Fujii,

Journal of Advanced Mechanical Design, Systems, and Manufacturing, Vol.15, No.4 (2021)

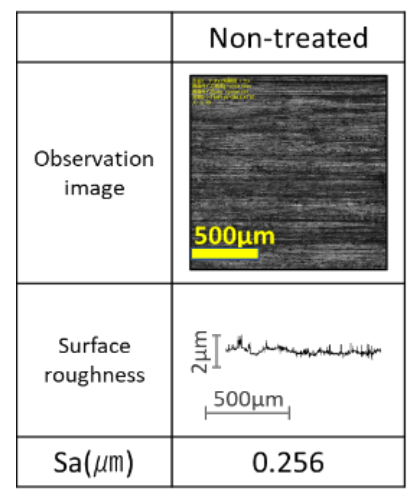

Fig. 4 Image observed through a laser microscope and surface roughness (non-treated)

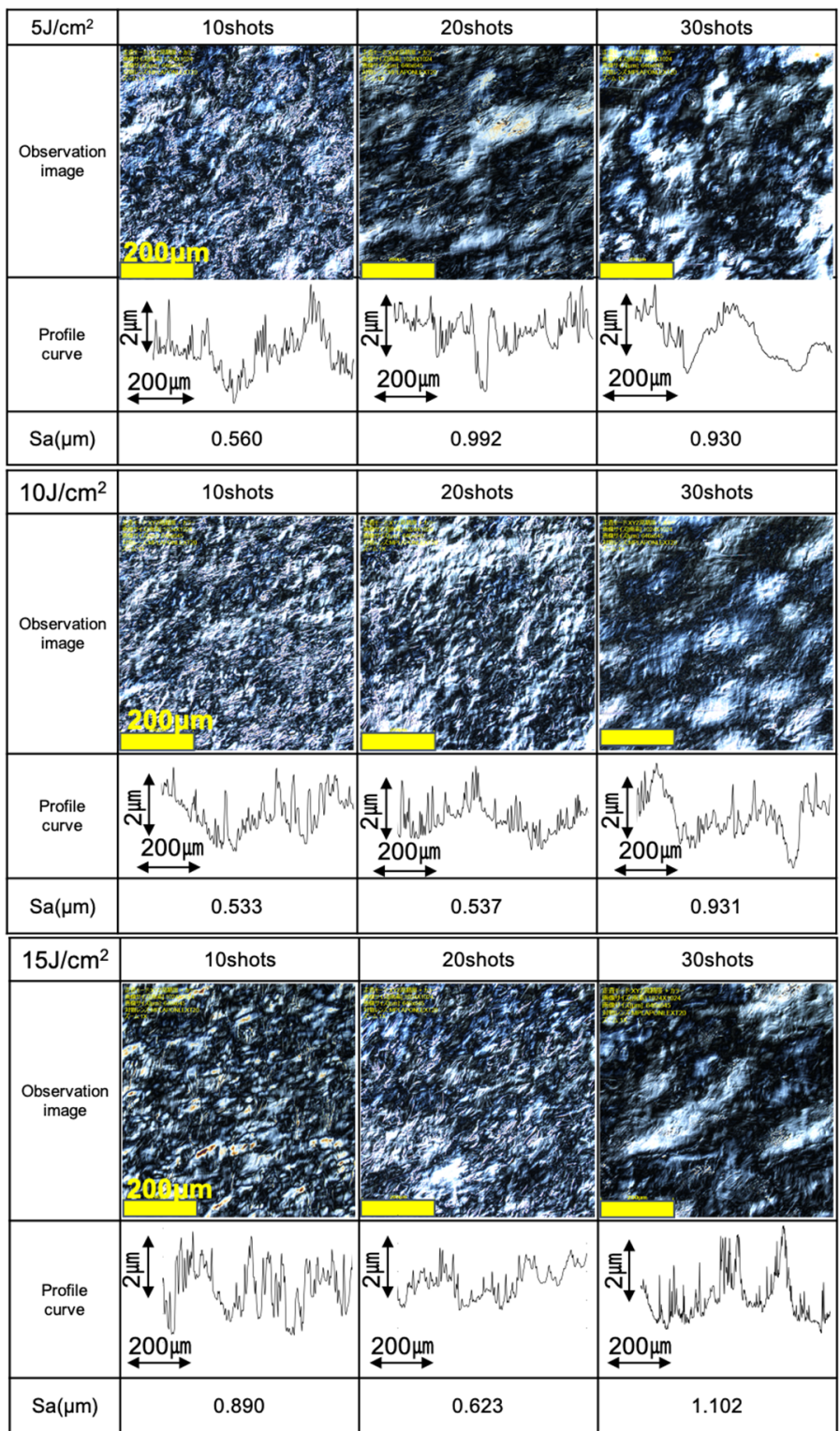

Fig. 5 Image observed through a laser microscope and surface roughness (large-area EB irradiation) 
Focusing on Al, the abundance ratio was less than $5 \%$ on the non-treated surface. However, it accounted for $20-30 \%$ on the surfaces of EB and plasma specimens. This increase is attributed to the removal of the organic matter layer on the adherend surface.

Focusing on $\mathrm{O}$, the value exceeded $50 \%$ for the $\mathrm{EB}$ and plasma surfaces. One of the reasons for this was that the abundance ratio of $\mathrm{Al}\left(\mathrm{Al}_{2} \mathrm{O}_{3}\right.$, aluminum oxide) as mentioned above increased. For the plasma surface, it was also presumed that oxygen was chemically bonded to the adherend surface by plasma under an atmospheric environment.

The analytical result of the atomic concentration on the adherend surface did not change significantly regardless of the irradiation conditions.

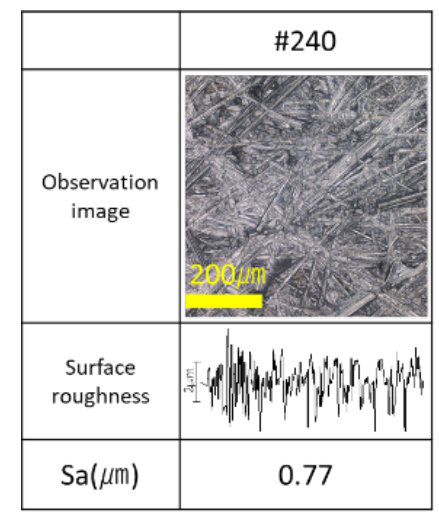

Fig. 6 Image observed through a laser microscope and surface roughness (emery paper)

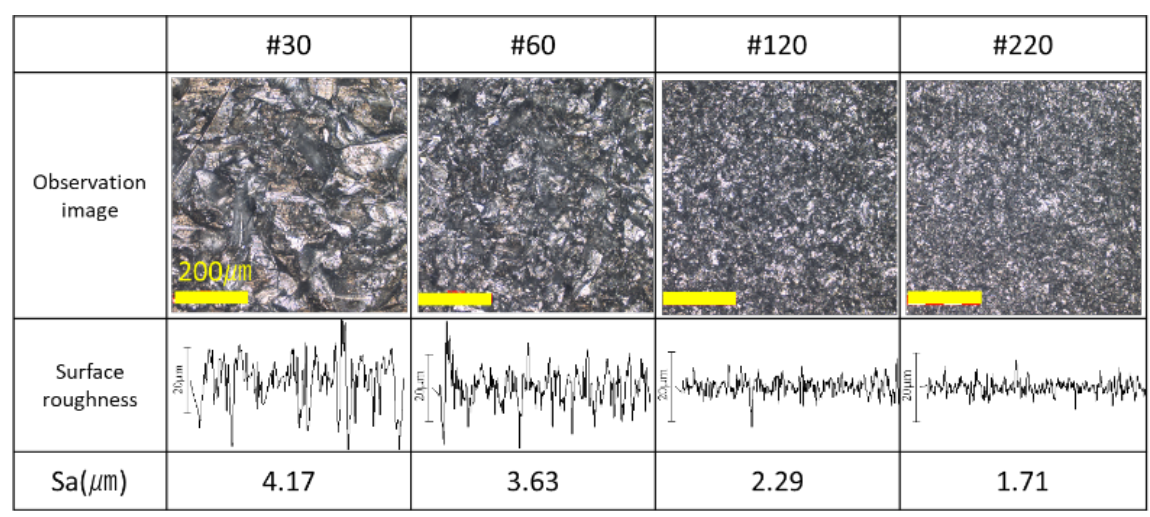

Fig. 7 Image observed through a laser microscope and surface roughness (shot blast)

Table 2 Atomic concentration (atomic \%)

\begin{tabular}{l|c|c|c}
\hline & C1s & O1s & Al2p \\
\hline Non-treated & 67.8 & 27.6 & 4.6 \\
\hline Plasma & 17.6 & 64.4 & 18.0 \\
\hline EB5J10S & 14.1 & 56.0 & 29.9 \\
\hline EB5J30S & 14.7 & 54.1 & 31.2 \\
\hline EB10J10S & 14.0 & 55.6 & 30.4 \\
\hline EB10J30S & 22.3 & 50.3 & 27.4 \\
\hline EB15J10S & 18.6 & 49.7 & 31.7 \\
\hline EB15J30S & 14.3 & 53.7 & 32.0 \\
\hline
\end{tabular}




\subsection{Wettability of adherend surface}

Wettability of the adherend surface is an important parameter for judging the adhesive strength. Therefore, the contact angle of water on the adherend surface was measured, which is shown in Figure 8. The ordinate shows the names of the treatments, while the abscissa represents the contact angle. It is seen that the contact angle of treated specimens, except for the EP surface, decreased compared with that of the untreated surface. In other words, the wettability of the adherend surface increased after the treatments. Differences in contact angle were not observed among the EB, EP, and plasma surfaces. However, the reason for the improvement in wettability was considered to be different for the three treated surfaces. It was considered that the SB surface had an improved wettability due to the change in the surface profile. For EB and plasma surfaces, it was attributed to the removal of the organic matter on the adherend surface and increase of the abundance ratio of $\mathrm{O}$. Furthermore, in EB, it was considered that the wettability was improved by the change in the surface profile. However, the reason why the wettability of the EP surface was not improved was considered to be that the linear polishing marks hindered the wetting and spreading of water.

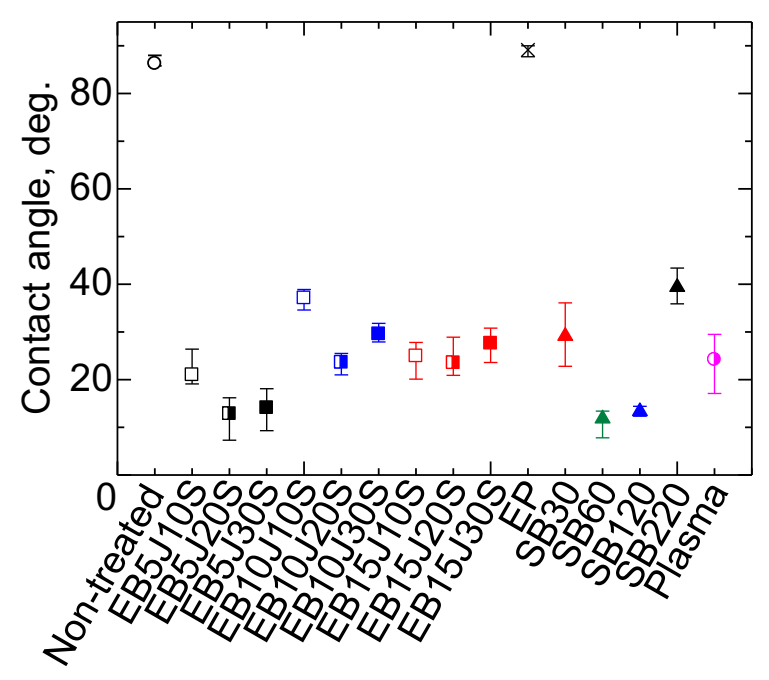

Fig. 8 Contact angle of adherend surface after surface treatments

\section{Result of tensile tests}

\subsection{Tensile strength}

Figure 9 shows the results of the tensile test. The ordinate represents the rupture stress (tensile load at rupture/adhesion area), while the abscissa represents the strain at rupture. It was observed that the rupture stress was improved by every surface treatment.

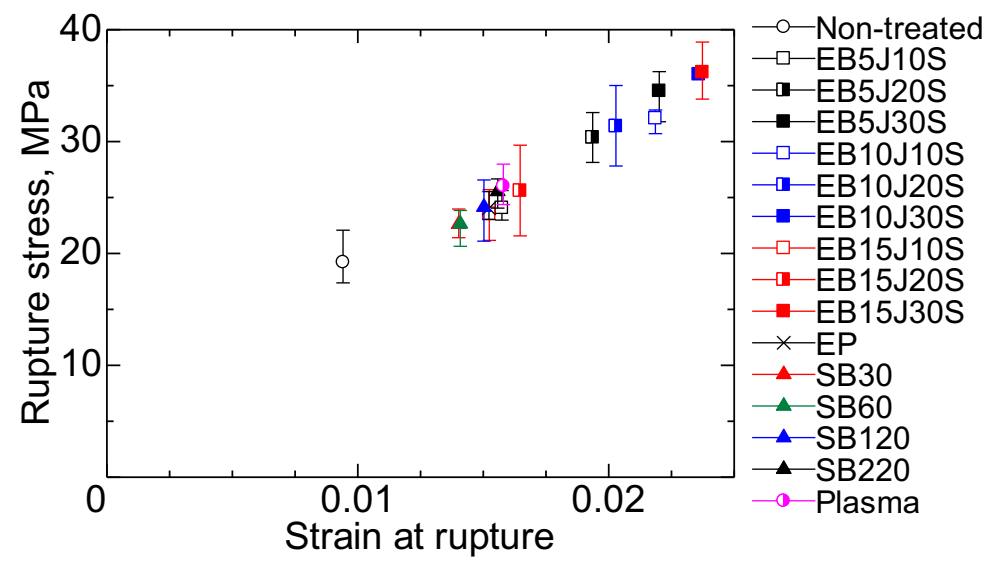

Fig. 9 Rupture stress and strain obtained from tensile test 
The specimen treated by large-area EB irradiation exhibited the maximum rupture stress. The maximum rupture stress of the EB specimen was approximately 1.9 times larger than that of the non-treated specimen. In addition, the maximum strain of the EB specimen was about 2.5 times larger than that of the non-treated specimen. The rupture stress improved as the shot number of EB irradiations increased. The inference of changes in the energy density on the rupture stress was not significant. However, considering the relationship between the shot number of EB irradiations and the rupture stress, it can be observed that the specimen irradiated with an energy density smaller than $15 \mathrm{~J} / \mathrm{cm}^{2}$ showed a linear relationship, and the variation in rupture stress was smaller.

For the EP and SB specimens having rough surfaces, their rupture stress was improved. The rupture stress of the SB specimen was about 1.3 times larger than that of the non-treated specimen. The inference of surface profiles was considered. However, although the surface roughness was different among the specimens, there was not much difference in the rupture stress. For the plasma specimen with a chemically modified surface, the rupture stress was improved even though the roughness did not change. The rupture stress of the plasma specimen was approximately 1.4 times larger than that of the non-treated specimen.

\subsection{Fracture surface and morphology}

Figures 10-14 show the images of the fracture surfaces of the non-treated, EB, EP, SB and plasma specimens obtained by the laser microscope, respectively. As generally known, it was considered that the fracture of the adhesive joint initiated from the end of the adherend in this study as well.

It was observed that interface fracture occurred mainly in the non-treated specimen. It is well known that the strength of an adhesive joint with interface fracture is lower than that with cohesion fracture. In the EB specimen, both interface and cohesion fractures were observed, and the fracture surface was not seen to be symmetric. The crack propagation in the fracture surface of this specimen was more complicated than that of the other treated specimens. As the number of shots

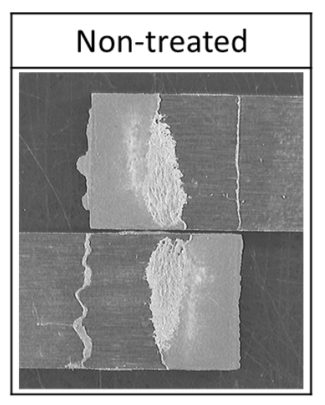

Fig. 10 Image of fracture surface observed by laser microscope (non-treated)

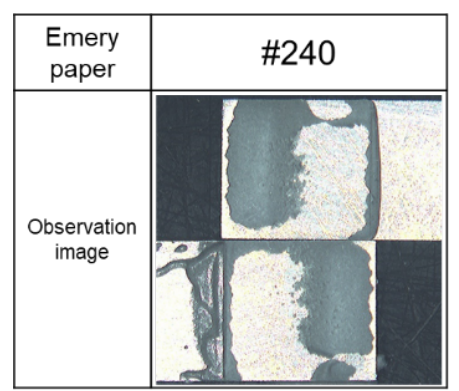

Fig. 12 Image of the fracture surface observed through a laser microscope (Emery paper)
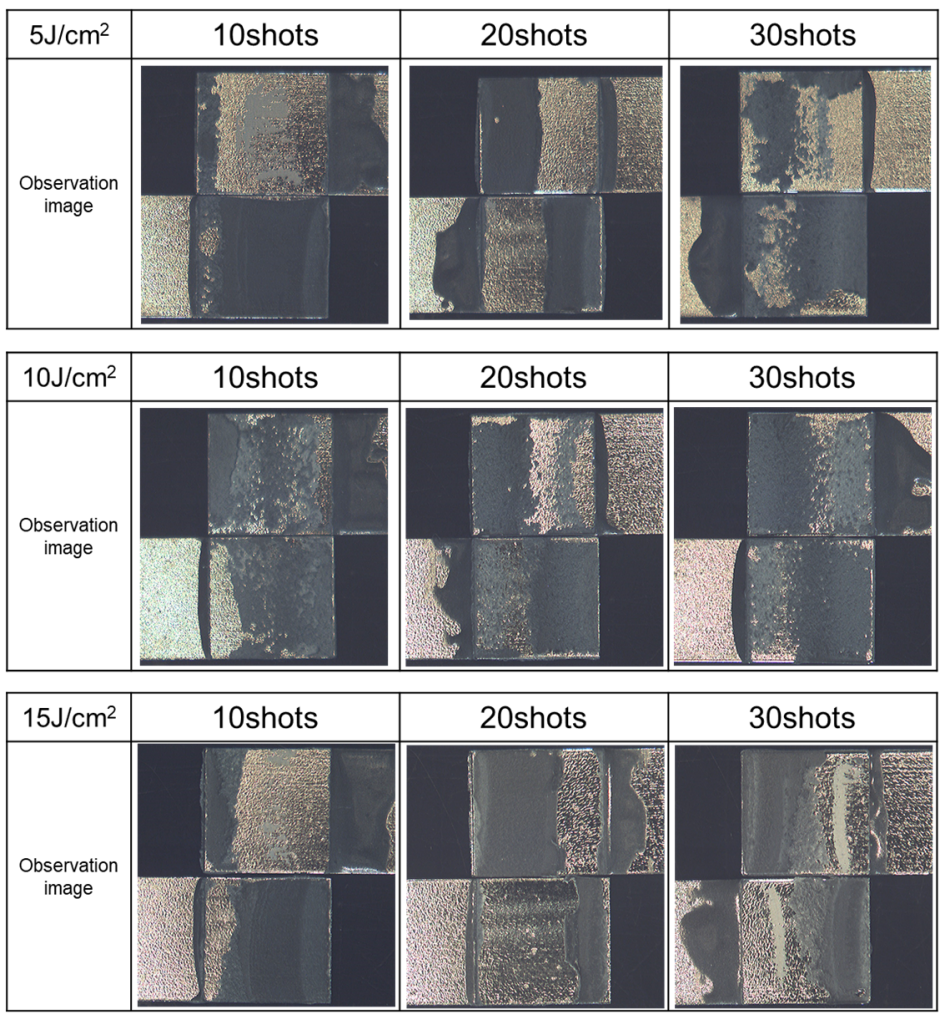

Fig. 11 Image of the fracture surface observed through a laser microscope (large-area EB irradiation) 


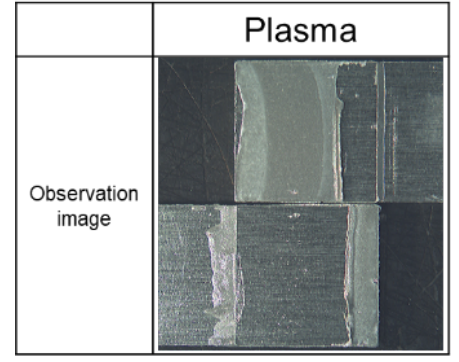

Fig. 14 Image of the fracture surface observed through a laser microscope (plasma)

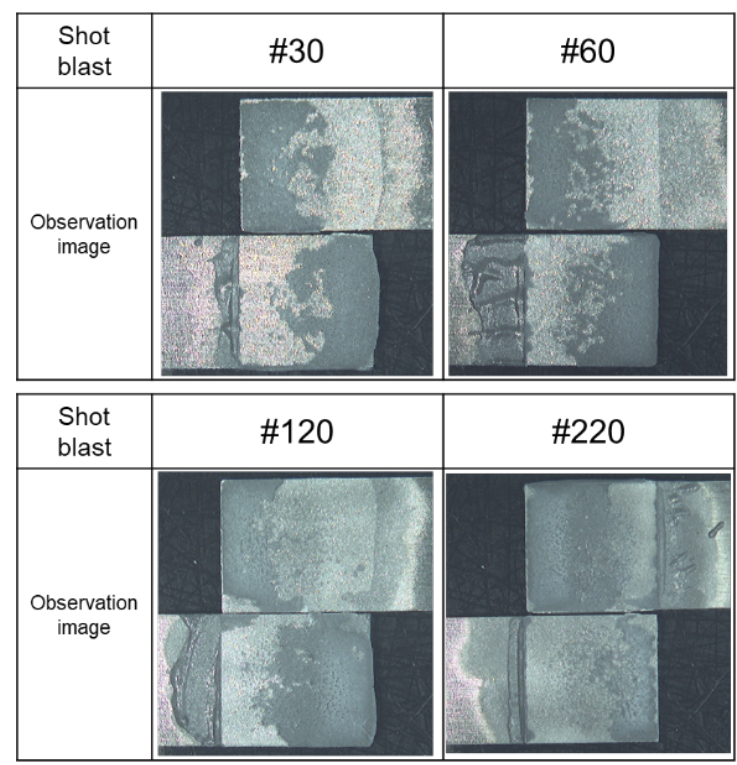

Fig. 13 Image of the fracture surface observed through a laser microscope (shot blast)

increased, more areas of cohesive failure were observed in the specimen.

For the EP and SB specimens, it could be seen that the cohesion fracture was partly accrued in addition to interface fracture, and the fracture surfaces were seen to be symmetric.

For the plasma specimen, there were very few cohesion fractures on the interfaces. However, the fracture crosssection did not show a symmetric surface.

\section{Discussions}

\subsection{Effect of surface roughness}

Figure 15 shows the relationship between the surface roughness and rupture stress of the non-treated, EP, and SB specimens. From this figure, a tendency to have an extremal value between $1 \mu \mathrm{m}$ and $1.5 \mu \mathrm{m}$ can be seen. Regarding the fracture morphology, it seems that the interface fracture transforms into the cohesive fracture around this extremal value. This may be because the roughness of the surface increased the stress inside the adhesive layer near the roughness, and the crack propagation shifted to the inside of the adhesive layer instead of the interface. Thus, the strength of the adhesive joint was improved. However, an excessive local stress easily causes cohesive fracture and leads to a decrease in the joint strength. Figure 16 shows the relationship between the surface roughness and the rupture stress of the EB and plasma specimens. Although differences in the surface of the adherend in terms of atomic concentration were seen, the rupture $\mathrm{s}$

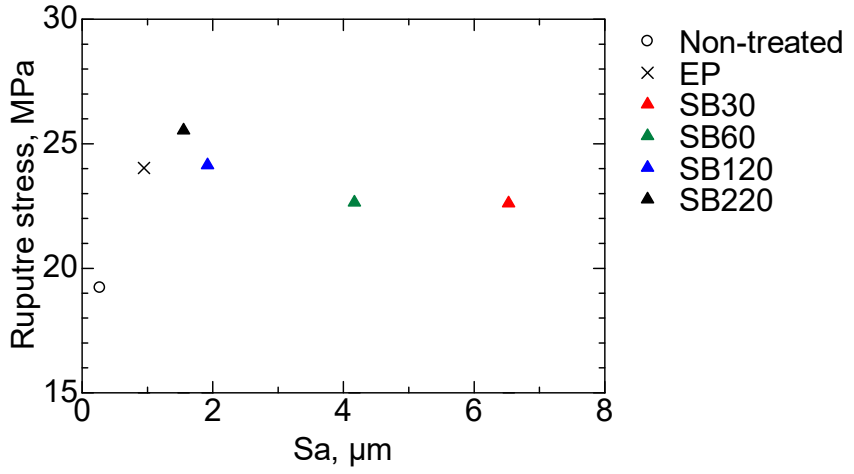

Fig. 15 Inference of surface roughness on rupture stress (EP and SB specimens)

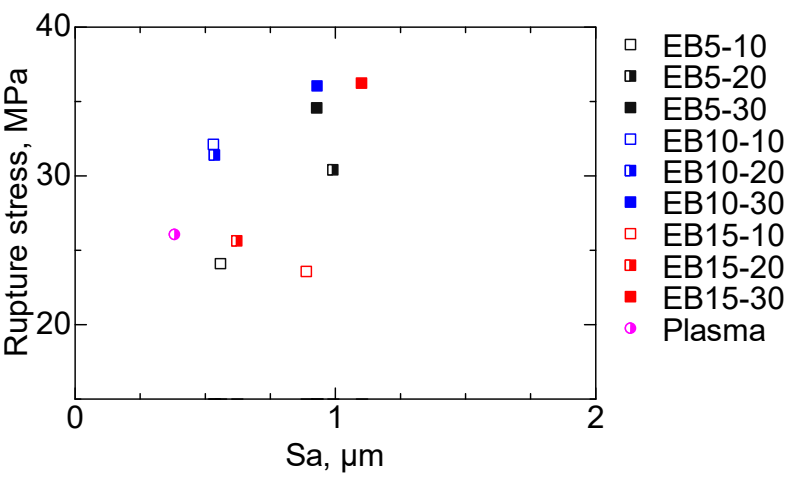

Fig. 16 Inference of surface roughness on rupture stress (EB and plasma specimens) 
tress tended to increase toward $1.5 \mu \mathrm{m}$, similar to that shown in Figure 15 . Under the energy density of $15 \mathrm{~J} / \mathrm{cm}^{2}$, no dependency on the number of shots was observed. Therefore, an energy density of $15 \mathrm{~J} / \mathrm{cm}^{2}$ or less was considered to be good for stable surface formation.

\subsection{Effects of wettability and atomic concentration}

The wettability of the EP and SB surfaces were significantly different (Fig. 8). However, the results of fracture surface observation showed no significant difference between them. Since the wettability was improved in the SB surface, it can be expected that the true contact area between the adherend and the adhesive was improved. However, since the fracture surface had a symmetrical appearance, it was presumed that the adhesion between them did not improve. Therefore, it was considered that the mechanism discussed previously was the main factor affecting the joint strength.

On the contrary, in the plasma specimen, the tensile strength of the adhesive joint was improved despite the interface fracture. From the results of surface analysis by XPS (Table 2), a large amount of $\mathrm{O}$ was detected on the plasma surface. It is generally known that oxides such as $\mathrm{Al}_{2} \mathrm{O}_{3}$ contain hydroxy groups. It is considered that such a functional group on the adherend surface improved the adhesive force. Thus, the fracture surface condition, such as symmetry, was changed.

For the EB specimen, the cohesion fracture and asymmetric surface were observed. This asymmetric property became stronger as the shot number increased, which might have also increased the chemical. The EB surface also had a surface shape suitable for improving the joint strength because the increase in surface roughness leads to an increase in the adhesion surface area. As a result, the EB specimen exhibited an improved joint strength owing to the synergistic effect of mechanical and chemical factors.

\section{Conclusions}

The tensile strengths of single lap joints were measured to investigate the influence of large-area EB irradiation and other surface treatments on the strength characterization of the adhesive joint. The effect of large-area EB irradiation on the adherend surface was clarified. The results obtained from this study are as follows:

(1) The specimen treated with large-area EB irradiation exhibited the maximum strength. It was found that the tensile strength of this specimen treated increased as the shot number increased.

(2) The maximum rupture stress of the EB specimen was approximately 1.9 times larger than that of the non-treated specimen. In addition, the maximum strain of the EB specimen was about 2.5 times larger than that of the nontreated specimen.

(3) Interface and cohesion fractures were observed in the specimen treated by large-area EB irradiation, and the fracture surface was unsymmetric.

\section{Acknowledgements}

We are grateful to MARUBUN CORPORATION for supplying the atmospheric pressure plasma device and for their valuable advice regarding the plasma treatment conditions.

\section{References}

da Silva, L. F. M., Adams, R. D., Joint strength predictions for adhesive joints to be used over a wide temperature range, International Journal of Adhesion and Adhesives, Vol.27, No.5(2007), pp.362-379. DOI: 10.1016/j.ijadhadh.2006.09.007

Ghumatkar, A., Budhe, S., Sekhar, R., Banea, M. D., de Barros, S., Influence of adherend surface roughness on the adhesive bond strength, Latin American Journal of solids and structures, Vol.13, No.13(2016), DOI: 10.1590/167978253066.

Harris, A. F., Beevers, A., The effects of grit-blasting on surface properties for adhesion, International Journal of adhesion. and adhesives, Vol.19, No.6 (1999), pp.445-452. DOI: 10.1016/S0143-7496(98)00061-X

Misukure, W., Kosugi, M., Yoshida, S., Kawabata, Y., Sugibayashi, T., Evaluation of the adhesive strength on high viscosity cyanoacrylate adhesive resin using developed interfacial area ratio of the scale-limited surface (Sdr), Journal of the Adhesion Society of Japan: Adhesion, Vol.55, No.1 (2019), pp.20-55. DOI: 10.11618/adhesion.55.20 
Molitor, P., Barron, V., Young, T., Surface treatment of titanium for adhesive bonding to polymer composites: a review, International Journal of Adhesion and Adhesives, Vol.21, No.2 (2001), pp.129-136. DOI: 10.1016/S01437496(00)00044-0

Sakata, O., Usui, Y., Shimamoto, M., Kohno, K., Kinoshita, Y., Miyashita, K., Influence of profile's shape of adherend surface on strength of adhesive bonding, Journal of the Japan Society of Precision Engineering, Vol.64, No.10 (1998), pp.1492-1496.

Sawa, T., Liu, J., Nakano, K., Tanaka, J., A two-dimensional stress analysis of single-lap adhesive joints of dissimilar. adherends subjected to tensile loads, Journal of Adhesion Science and Technology, Vol.14, No.1 (2000), pp.43-66 DOI: $10.1163 / 156856100742104$.

Shinonaga, T., Okada, A., Liu, H., Kimura, M., Magnetic fixtures for enhancement of smoothing effect by electron beam melting, Journal of materials processing technology, Vol.254, (2018), pp.229-237. DOI: 10.1016/j.jmatprotec.2017.11.024

Uno, Y., Okada, A., Uemura, K., Raharjo, P., Furukawa, T., Karato, K., High-efficiency finishing process for metal mold. by large-area electron beam irradiation, Precision Engineering, Vol.29, No.4 (2005), pp.449-455. DOI: 10.1016/j.precisioneng.2004.12.005 